\title{
INTRINSIC BRIGHTNESS TEMPERATURE OF COMPACT RADIO SOURCES AT $86 \mathrm{GHZ}$
}

\author{
SANG-SUNG LEE \\ Korea Astronomy and Space Science Institute, 776 Daedukdae-ro, Yusong, Daejon 305-348, Korea \\ E-mail : sslee@kasi.re.kr \\ (Received September 13, 2013; Revised October 29, 2013; Accepted November 4, 2013)
}

\begin{abstract}
We present results on the intrinsic brightness temperature of a sample of compact radio sources observed at $86 \mathrm{GHz}$ using the Global Millimeter VLBI Array. We use the observed brightness temperatures at $86 \mathrm{GHz}$ and the observed superluminal motions at $15 \mathrm{GHz}$ for the sample in order to constrain the characteristic intrinsic brightness temperature of the sample. With a statistical method for studying the intrinsic brightness temperatures of innermost jet cores of compact radio sources, assuming that all sources have the same intrinsic brightness temperature and the viewing angles of their jets are around the critical value for the maximal apparent speed, we find that sources in the sample have a characteristic intrinsic brightness temperature, $T_{0}=4.8_{-1.5}^{+2.6} \times 10^{9} \mathrm{~K}$, which is lower than the equipartition temperature for the condition that the particle energy equals to the magnetic field energy. Our results suggest that the VLBI cores seen at $86 \mathrm{GHz}$ may be representing a jet region where the magnetic field energy dominates the total energy in the jet.
\end{abstract}

Key words : Galaxies: nuclei — quasars: relativistic jets — radio: galaxies

\section{INTRODUCTION}

Compact radio sources are generally defined as radio sources whose flux density at an intermediate radio frequency, e.g., $\sim 1 \mathrm{GHz}$, is dominated by the emission of a single bright region within $\sim 1 \mathrm{kpc}$ in size (Blandford \& Königl 1979). Compact radio sources usually have flat radio spectra and exhibit pronounced radio variability. Moreover, due to the lager ratio of optical to radio flux of the compact radio sources than of steep-spectrum sources, these objects have been easily identified.

Radio emission from parsec-scale jets in compact radio sources consists of optically thin synchrotron emission, and characterized by their spectral and polarization properties and significant inverse-Compton emission (see Marscher 1990; Hughes \& Miller 1991). The flat spectrum of the radio emission is generally interpreted as due to superposition of incoherent synchrotron radiation from a non-thermal distribution of relativistic electrons located in several distinct components (Kellermann \& Pauliny-Toth 1969; Marscher 1995). These components form the innermost compact structure, the compact jet base at sub-parsec scales, and the bright emission regions at parsec scales of the jet. The physical processes of the formation of inner jets that connect the nucleus to the observed radio jet, their acceleration to relativistic speeds, and strong collimation to large scales (pc to $\mathrm{kpc}$ ) have been extensively investigated but remain poorly understood (e.g., Marscher 2006; Lobanov \& Zensus 2006; Lobanov 2007).
Readhead (1994) suggested that parsec-scale jets are in an equipartition condition that the energy density in relativistic particles is equal to that in magnetic fields (Burbidge \& Burbidge 1957). He found that the radio sources may radiate at an equipartition brightness temperature around $5 \times 10^{10} \mathrm{~K}$ in most circumstances. Recent studies have shown that extended radio lobes are indeed at equipartition (Croston et al. 2005 ), and the relativistic jets are at equipartition in their median-low state (Homan et al. 2006). However when the relativistic jets are in their maximum state, the brightness temperatures are a factor of 4 larger, implying the energy in their radiating particle is $\geq 10^{5}$ times larger than the energy in magnetic fields, based on the relation of the energy and brightness temperature as proposed in Readhead (1994): $\eta=u_{p} / u_{B}=$ $\left(T_{\text {eq }} / T_{\mathrm{b}}\right)^{-17 / 2}$, where $u_{p}, u_{B}$ are the energy densities of the radiating particles and the magnetic field, respectively, $T_{\mathrm{eq}} \simeq 5 \times 10^{10} \mathrm{~K}$ is the equipartition brightness temperature, and $T_{\mathrm{b}}$ is the observed brightness temperature. It is very difficult to measure intrinsic properties of extragalactic compact radio sources because the jets of compact radio sources are highly relativistic and therefore Doppler boosted (Blandford \& Königl 1979; Lind \& Blandford 1985). The physical aspects of the jet can be parameterized by the Lorentz factor $\gamma_{j}$, the intrinsic brightness temperature $T_{0}$, and the angle to the line of sight $\theta_{\mathrm{j}}$. From these intrinsic physical properties, one can calculate the Doppler factor $\delta$, the apparent jet speed $\beta_{\text {app }}$, and the observed brightness 
temperature $T_{\mathrm{b}}$ :

$$
\begin{gathered}
\delta=\frac{1}{\gamma_{\mathrm{j}}\left(1-\beta \cos \theta_{\mathrm{j}}\right)}, \\
\beta_{\mathrm{app}}=\frac{\beta \sin \theta_{\mathrm{j}}}{1-\beta \cos \theta_{\mathrm{j}}}, \\
T_{\mathrm{b}}=T_{0} \delta,
\end{gathered}
$$

where $\beta=\left(1-\gamma_{j}^{-2}\right)^{1 / 2}$ is the speed of jet in the rest frame of the source (units of $c$ ).

Observed brightness temperatures $\left(T_{\mathrm{b}}\right)$ of compact radio jets can be used to study the intrinsic physical properties of the relativistic jets. One application is to test accelerating and decelerating jet models (Marscher 1995) by investigating the change in the observed brightness temperatures measured at various radio frequencies (e.g., $2-86 \mathrm{GHz}$ ). Under equipartition conditions between jet particle and magnetic field energy densities, the position shift of the brightest jet components of VLBI images (VLBI cores) between two frequencies can be predicted (Lobanov 1998). The brightness temperatures in the rest frame of the sources and the predicted core shift should be able to test the inner jet models.

Another application is to obtain the intrinsic brightness temperatures of VLBI cores by using the observed brightness temperature $T_{\mathrm{b}}$ and the maximum apparent jet speed $\beta_{\text {app }}$. A method developed by Homan et al. (2006) was applied to the $2 \mathrm{~cm}$ survey data (Kellermann et al. 2004) in order to determine the intrinsic physical properties of prominent AGN (Cohen et al. 2007). They found that VLBI cores observed at $15 \mathrm{GHz}$ are near equipartition in their median-low state, resulting in intrinsic brightness temperatures of $T_{0}=3 \times 10^{10} \mathrm{~K}$.

The method can be applied to VLBI survey data at different frequencies, with the maximum apparent jet speeds taken from Kellermann et al. (2004), in order to constrain the intrinsic brightness temperature at these frequencies. In this paper, we combine the observed brightness temperature data from a global $86 \mathrm{GHz}$ VLBI imaging survey (Lee et al. 2008) and proper-motion data from the $2 \mathrm{~cm}$ VLBA survey. In Section 2, the theory and its application to the $2 \mathrm{~cm}$ VLBA survey are reviewed. In Section 3, we present our results using the $86 \mathrm{GHz}$ VLBI survey. In Section 4 , we discuss the interpretation of the intrinsic brightness temperatures at various frequencies.

\section{INTRINSIC BRIGHTNESS TEMPERA- TURE AT $15 \mathrm{GHz}$}

\subsection{Methodology}

Following Homan et al. (2006), we assume that a compact radio source contains an ideal relativistic jet, which is narrow and straight with no bends between the VLBI core and the jet components. Of course, some jets are not straight and $\theta_{\mathrm{j}}$ is not the same in

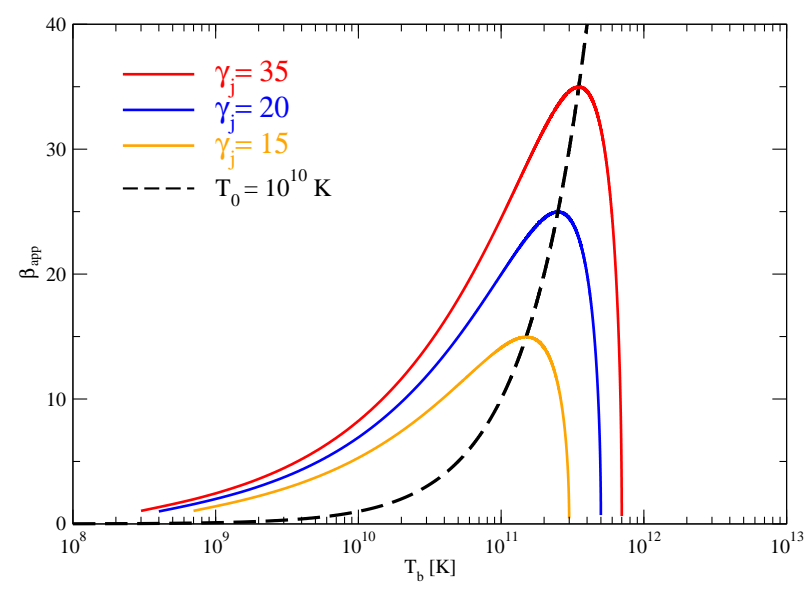

Fig. 1. - Plot of the apparent jet speed $\beta_{\text {app }}$ with the observed brightness temperature $T_{\mathrm{b}}$ for a single intrinsic brightness temperature of $T_{0}=1 \times 10^{10} \mathrm{~K}$ and several values of Lorentz factors $\gamma_{j}=15,20,35$. The dashed line represents where sources observed at the critical angle would lie on this plot. The solid line represents the possible apparent speeds of a $\gamma_{\mathrm{j}}$ source with intrinsic brightness temperature given by $T_{0}$.

the core and in the moving jet components. The celebrated example and evidence of the jet bending are found in 3C 279 (Homan et al. 2003; Abdo et al. 2010). However, as long as superluminal motion is observed, the motion must be close to the line of sight, and angular changes of the motion could be highly magnified due to projection effect. A jet with an intrinsic bend of only a few degrees could be observed as a right-anlge bending jet (Cohen et al. 2007).

In this case, we can also assume that the maximum speed of the jet component is the same as the speed of the jet flow through the jet core. The flow speed of the jet is usually different from the pattern speed of the jet in some low-luminosity sources. However, in those sources which are bright and straight, the pattern speeds are the same as the flow speeds. For simplicity, we make two further assumptions:

1. The intrinsic brightness temperature $T_{0}$ of all jets are the same, and

2. The viewing angels of their jets are around the critical value $\theta_{\mathrm{c}}=\arccos \beta$ for the maximal apparent speed at a given $\beta$.

Under the assumptions above, one can relate the observed brightness temperature to the maximum jet speed:

$$
\delta \simeq \beta_{\mathrm{app}}
$$

and

$$
T_{\mathrm{b}} \simeq \beta_{\mathrm{app}} T_{0} .
$$

This resultant simple relation between the observed brightness temperature and the apparent maximum jet speed is illustrated in Fig. 1. From Eqs. 1 and 2, 
one can also relate the apparent jet speed $\beta_{\text {app }}$ to the Doppler factor $\delta$ and the Lorentz factor $\gamma_{\mathrm{j}}$ :

$$
\beta_{\mathrm{app}}=\sqrt{\left(\delta \gamma_{\mathrm{j}} \beta\right)^{2}-\left(\delta \gamma_{\mathrm{j}}-1\right)^{2}}
$$

This relation is illustrated as three solid lines in Figure 1 , for the maximum and minimum possible Doppler factors $\delta_{\max }=1 / \gamma_{\mathrm{j}}$ and $\delta_{\min }=1 /\left(\gamma_{\mathrm{j}}-\sqrt{\gamma_{\mathrm{j}}^{2}-1}\right)$. The lines show actually the apparent speeds as functions of $T_{\mathrm{b}}$ for jets with Lorentz factors of $\gamma_{\mathrm{j}}=15,20$, and 25. Homan et al. (2006) found that from the simulation of a relativistically beamed population of 1000 fictional compact radio sources with given $T_{0}$ and $\gamma_{\mathrm{j}}$, the dashed line divided the 1000 compact radio sources into two groups: one group of about 750 sources in the right and below, and another group of about 250 sources in the left and above. The viewing angles of 750 sources in the right and below are smaller than the critical angle, and their Doppler factors are large. Their proper motions are small due to their viewing angle.

\subsection{Application to $15 \mathrm{GHz}$ Data}

By combining the $2 \mathrm{~cm}$ VLBA survey data and the proper motion data of AGN jets, Homan et al. (2006) found an intrinsic brightness temperature of $T_{0} \simeq 3 \times 10^{10} \mathrm{~K}$ of the sources in their sample when the sources are in their median-low (25\%-median) brightness temperature state, that is for median brightness temperatures of a lower half sample of observed brightness temperaturs obtained from multi-epoch observations of individual AGN. This value for $T_{0}$ is close to the equipartition temperature under the condition that the particle energy equals to the magnetic field energy. However, for maximum observed brightness temperatures, they also found a characteristic intrinsic brightness temperature of $2 \times 10^{11} \mathrm{~K}$ of their sample, which is brighter than the equipartition temperature by a factor of 4 . This implies that, in the maximum brightness state, the energy in radiating particles exceeds the energy in the magnetic field by a factor of $\sim 10^{5}$. They suggest that at the innermost regions of the jet, injection or acceleration of particles should maintain the energy in the radiating particles dominant over the energy in the magnetic field.

\section{INTRINSIC BRIGHTNESS TEMPERA- TURE AT $86 \mathrm{GHz}$}

\subsection{A Global $86 \mathrm{GHz}$ VLBI Survey Data}

In an attempt to investigate intrinsic brightness temperature for sources observed at $86 \mathrm{GHz}$, we used the observed brightness temperatures of VLBI cores at $86 \mathrm{GHz}$ from a large global $86 \mathrm{GHz}$ VLBI survey of compact radio sources (Lee et al. 2008). The survey data consist of total intensity images with a typical image FWHM restoring beam of approximately $40 \mu$ as. This corresponds to a scale of $<0.1$ parsecs at typical redshifts $\mathrm{z} \cong 1$ for our sample AGNs. We used Gaussian fit to the VLBI core component of each jet to determine a rest-frame core brightness temperature $T_{\mathrm{b}}$ for each jet according to

$$
T_{\mathrm{b}}=1.22 \times 10^{12} \frac{S_{\mathrm{tot}}}{d^{2} \nu^{2}}(1+z) \mathrm{K}
$$

where $S_{\text {tot }}$ is the fitted core flux density in Janskys at $\nu=86 \mathrm{GHz}, d$ is the FWHM dimensions of the fitted circular core components in milliarcseconds. In determining the FWHM $d$ of a core component, the resolution limit of the determination was taken into account. So, the minimum resolvable size of a component in an image is given by

$$
d_{\min }=\frac{2^{1+\beta / 2}}{\pi}\left[\pi a b \ln 2 \ln \frac{S N R}{S N R-1}\right]^{1 / 2}
$$

where $a$ and $b$ are the axes of the restoring beam, $S N R$ is the signal-to-noise ratio, and $\beta$ is a weighting function, which is 0 for natural weighting or 2 for uniform weighting. When $d<d_{\min }$, the uncertainties should be estimated with $d=d_{\text {min. }}$. If $d<d_{\text {min }}$, then the lower limit of $T_{\mathrm{b}}$ is obtained with $d=d_{\min }$.

\subsection{VLBA Data}

Since there are no reliable measurements of the apparent jet speeds at $86 \mathrm{GHz}$, we used the apparent jet speeds from the $2 \mathrm{~cm}$ survey and the MOJAVE survey (Kellermann et al. 2004; Lister et al. 2009, 2013), thereby assuming that the apparent jet speeds at 15 and $86 \mathrm{GHz}$ are similar each other. We selected the fastest proper motions for each source from the MOJAVE survey (Lister et al. 2009, 2013), assuming that the speeds are maximum values of individual sources. For some sources whose proper motions are not available in the MOJAVE survey, we used the apparent speeds from the 2cm survey (Kellermann et al. 2004), and we only considered those speeds that are ranked as "excellent" (E) or "good" (G) by their criteria. We found that apparent jet speeds at $15 \mathrm{GHz}$ are available for 98 of the sources in the $86 \mathrm{GHz}$ survey,

In order to constrain the characteristic intrinsic brightness temperature at $15 \mathrm{GHz}$ for our sample, we obtained the maximum observed brightness temperatures $\left(T_{\mathrm{b}}^{15 \mathrm{GHz}, \max }\right)$ and the $25 \%$-median brightness temperatures $\left(T_{\mathrm{b}}^{15 \mathrm{GHz}, 25 \% \text { med }}\right)$ from Kovalev et al. (2005). Table 1 lists the observed brightness temperatures at 15 and $86 \mathrm{GHz}$ for the selected target sources, with the optical class and redshift obtained from Véron-Cetty \& Véron (2006). The final sample contains 98 sources, consisting of 7 galaxies, 20 BL Lac objects, 70 quasars, and one unidentified source according to the optical class. 
Table 1.

Observed brightness temperatures

\begin{tabular}{|c|c|c|c|c|c|c|}
\hline Name & Type & $z$ & $\begin{array}{c}\beta_{\mathrm{app}} \\
{[c]}\end{array}$ & $\begin{array}{c}T_{\mathrm{b}}^{15 \mathrm{GHz}, \max } \\
{[\mathrm{K}]}\end{array}$ & $\begin{array}{l}T_{\mathrm{b}}^{15 \mathrm{GHz}, 25 \% \text { med }} \\
{[\mathrm{K}]}\end{array}$ & $\begin{array}{l}T_{\mathrm{b}}^{86 \mathrm{GHz}} \\
{[\mathrm{K}]}\end{array}$ \\
\hline $0003-066$ & B & 0.347 & $8.39 \pm 0.39$ & $>4.60 \mathrm{e}+11$ & $8.07 \mathrm{e}+10$ & $>2.60 \mathrm{e}+10$ \\
\hline $0007+106$ & $\mathrm{G}$ & 0.089 & $1.20 \pm 0.07$ & $9.05 \mathrm{e}+11$ & $1.82 \mathrm{e}+11$ & $>1.20 \mathrm{e}+10$ \\
\hline $0016+731$ & $\mathrm{Q}$ & 1.781 & $8.23 \pm 0.34$ & $>7.24 \mathrm{e}+12$ & $1.14 \mathrm{e}+11$ & $>1.80 \mathrm{e}+11$ \\
\hline $0048-097$ & $\mathrm{~B}$ & $\ldots$ & $0.00 \pm 0.00$ & $>7.31 \mathrm{e}+12$ & $>2.44 \mathrm{e}+11$ & $2.50 \mathrm{e}+10$ \\
\hline $0106+013$ & Q & 2.107 & $24.40 \pm 3.90$ & $>3.87 \mathrm{e}+12$ & $8.83 \mathrm{e}+11$ & $1.70 \mathrm{e}+11$ \\
\hline $0119+041$ & $\mathrm{Q}$ & 0.637 & $0.50 \pm 1.60$ & $>1.86 \mathrm{e}+11$ & $1.04 \mathrm{e}+11$ & $>1.40 \mathrm{e}+10$ \\
\hline $0119+115$ & $\mathrm{Q}$ & 0.570 & $18.58 \pm 0.82$ & $3.37 \mathrm{e}+11$ & $1.50 \mathrm{e}+11$ & $>5.90 \mathrm{e}+10$ \\
\hline $0133+476$ & $\mathrm{Q}$ & 0.859 & $15.40 \pm 1.20$ & $>1.91 \mathrm{e}+13$ & $7.14 \mathrm{e}+11$ & $2.40 \mathrm{e}+11$ \\
\hline $0149+218$ & $\mathrm{Q}$ & 1.32 & $18.40 \pm 1.90$ & $>1.40 \mathrm{e}+12$ & $5.80 \mathrm{e}+11$ & $5.10 \mathrm{e}+10$ \\
\hline $0202+149$ & $\mathrm{Q}$ & 0.405 & $15.88 \pm 0.75$ & $>1.53 \mathrm{e}+12$ & $1.82 \mathrm{e}+11$ & $>4.80 \mathrm{e}+10$ \\
\hline $0202+319$ & $\mathrm{Q}$ & 1.466 & $10.10 \pm 1.00$ & $>2.67 \mathrm{e}+12$ & $1.10 \mathrm{e}+12$ & $7.90 \mathrm{e}+10$ \\
\hline $0212+735$ & $\mathrm{Q}$ & 2.367 & $6.58 \pm 0.18$ & $1.08 \mathrm{e}+12$ & $2.00 \mathrm{e}+11$ & $>2.70 \mathrm{e}+09$ \\
\hline $0224+671$ & $\mathrm{Q}$ & 0.523 & $13.69 \pm 0.56$ & $>3.94 \mathrm{e}+11$ & $>3.94 \mathrm{e}+11$ & $>6.60 \mathrm{e}+10$ \\
\hline $0234+285$ & $\mathrm{Q}$ & 1.207 & $22.00 \pm 1.10$ & $>3.83 \mathrm{e}+12$ & $>4.51 \mathrm{e}+11$ & $2.40 \mathrm{e}+11$ \\
\hline $0238-084$ & $\mathrm{G}$ & 0.005 & $0.39 \pm 0.02$ & $>3.83 \mathrm{e}+12$ & $>4.51 \mathrm{e}+11$ & $>2.20 \mathrm{e}+10$ \\
\hline $0300+470$ & B & & $0.00 \pm 0.00$ & $>8.46 \mathrm{e}+11$ & $>8.46 \mathrm{e}+11$ & $2.90 \mathrm{e}+10$ \\
\hline $0316+413$ & $\mathrm{G}$ & 0.017 & $0.29 \pm 0.02$ & $1.45 \mathrm{e}+11$ & $1.45 \mathrm{e}+11$ & $4.70 \mathrm{e}+10$ \\
\hline $0333+321$ & $\mathrm{Q}$ & 1.263 & $13.05 \pm 0.16$ & $>5.11 \mathrm{e}+12$ & $2.64 \mathrm{e}+11$ & $1.40 \mathrm{e}+11$ \\
\hline $0336-019$ & $\mathrm{Q}$ & 0.852 & $24.40 \pm 1.60$ & $>3.24 \mathrm{e}+12$ & $4.51 \mathrm{e}+11$ & $5.60 \mathrm{e}+10$ \\
\hline $0355+508$ & $\mathrm{Q}$ & 1.52 & $1.90 \pm 1.60$ & $>9.77 \mathrm{e}+11$ & $>6.59 \mathrm{e}+11$ & $1.20 \mathrm{e}+11$ \\
\hline $0415+379$ & $\mathrm{G}$ & 0.049 & $8.14 \pm 0.31$ & $>7.63 \mathrm{e}+11$ & $1.56 \mathrm{e}+11$ & $4.10 \mathrm{e}+10$ \\
\hline $0420+022$ & Q & 2.277 & $8.51 \pm 0.98$ & $5.62 \mathrm{e}+11$ & $3.02 \mathrm{e}+11$ & $6.70 \mathrm{e}+10$ \\
\hline $0420-014$ & $\mathrm{Q}$ & 0.915 & $5.76 \pm 0.59$ & $>5.18 \mathrm{e}+13$ & $>2.12 \mathrm{e}+12$ & $1.90 \mathrm{e}+11$ \\
\hline $0422+004$ & $\mathrm{~B}$ & 0.310 & $0.39 \pm 0.19$ & $>1.38 \mathrm{e}+12$ & $>1.36 \mathrm{e}+12$ & $>5.60 \mathrm{e}+10$ \\
\hline $0430+052$ & G & 0.033 & $6.43 \pm 0.24$ & $>9.19 \mathrm{e}+11$ & $>1.04 \mathrm{e}+11$ & $9.80 \mathrm{e}+10$ \\
\hline $0440-003$ & $\mathrm{Q}$ & 0.844 & $0.59 \pm 0.15$ & $2.31 \mathrm{e}+11$ & $6.87 \mathrm{e}+10$ & $>5.20 \mathrm{e}+10$ \\
\hline $0458-020$ & $\mathrm{Q}$ & 2.291 & $13.56 \pm 0.82$ & $1.82 \mathrm{e}+12$ & $8.17 \mathrm{e}+11$ & $1.20 \mathrm{e}+11$ \\
\hline $0528+134$ & $\mathrm{Q}$ & 2.07 & $17.33 \pm 0.55$ & $>2.06 \mathrm{e}+13$ & $1.11 \mathrm{e}+12$ & $>2.50 \mathrm{e}+11$ \\
\hline $0529+075$ & $\mathrm{Q}$ & 1.254 & $18.00 \pm 1.10$ & $>1.91 \mathrm{e}+10$ & $>1.48 \mathrm{e}+10$ & $>1.30 \mathrm{e}+11$ \\
\hline $0552+398$ & $\mathrm{Q}$ & 2.363 & $1.63 \pm 0.10$ & $1.16 \mathrm{e}+12$ & $6.01 \mathrm{e}+11$ & $2.20 \mathrm{e}+11$ \\
\hline $0607-157$ & $\mathrm{Q}$ & 0.324 & $1.20 \pm 0.83$ & $>1.08 \mathrm{e}+13$ & $1.49 \mathrm{e}+12$ & $2.90 \mathrm{e}+10$ \\
\hline $0642+449$ & $\mathrm{Q}$ & 3.408 & $8.52 \pm 0.41$ & $>2.84 \mathrm{e}+13$ & $4.32 \mathrm{e}+12$ & $1.60 \mathrm{e}+11$ \\
\hline $0707+476$ & $\mathrm{Q}$ & 1.292 & $-2.80 \pm 1.70$ & $5.33 \mathrm{e}+11$ & $2.67 \mathrm{e}+11$ & $7.10 \mathrm{e}+10$ \\
\hline $0716+714$ & $\mathrm{~B}$ & $\ldots$ & $0.00 \pm 0.00$ & $>1.85 \mathrm{e}+13$ & $>3.60 \mathrm{e}+11$ & $3.60 \mathrm{e}+11$ \\
\hline $0727-115$ & $\mathrm{Q}$ & 1.591 & $31.20 \pm 0.60$ & $>9.93 \mathrm{e}+12$ & $>9.61 \mathrm{e}+11$ & $1.40 \mathrm{e}+10$ \\
\hline $0735+178$ & $\mathrm{~B}$ & 0.424 & $5.05 \pm 0.61$ & $>6.99 \mathrm{e}+11$ & $>1.34 \mathrm{e}+11$ & $2.40 \mathrm{e}+10$ \\
\hline $0736+017$ & $\mathrm{Q}$ & 0.191 & $13.79 \pm 0.20$ & $>2.51 \mathrm{e}+12$ & $5.09 \mathrm{e}+11$ & $1.00 \mathrm{e}+11$ \\
\hline $0738+313$ & $\mathrm{Q}$ & 0.630 & $10.70 \pm 1.20$ & $>6.54 \mathrm{e}+11$ & $>7.37 \mathrm{e}+10$ & $3.60 \mathrm{e}+10$ \\
\hline $0748+126$ & $\mathrm{Q}$ & 0.889 & $14.57 \pm 0.56$ & $>2.66 \mathrm{e}+12$ & $9.06 \mathrm{e}+11$ & $1.60 \mathrm{e}+11$ \\
\hline $0804+499$ & $\mathrm{Q}$ & 1.432 & $1.17 \pm 0.23$ & $>2.94 \mathrm{e}+12$ & $1.04 \mathrm{e}+12$ & $6.20 \mathrm{e}+10$ \\
\hline $0814+425$ & $\mathrm{~B}$ & 0.530 & $0.00 \pm 0.00$ & $>1.40 \mathrm{e}+12$ & $>1.09 \mathrm{e}+11$ & $2.30 \mathrm{e}+10$ \\
\hline $0823+033$ & $\mathrm{~B}$ & 0.506 & $12.88 \pm 0.49$ & $>3.01 \mathrm{e}+12$ & $>5.29 \mathrm{e}+11$ & $5.00 \mathrm{e}+10$ \\
\hline $0827+243$ & $\mathrm{Q}$ & 0.941 & $19.80 \pm 1.30$ & $1.93 \mathrm{e}+12$ & $6.23 \mathrm{e}+11$ & $>2.10 \mathrm{e}+11$ \\
\hline $0836+710$ & $\mathrm{Q}$ & 2.218 & $21.10 \pm 0.77$ & $6.18 \mathrm{e}+12$ & $2.97 \mathrm{e}+11$ & $>1.70 \mathrm{e}+11$ \\
\hline $0850+581$ & $\mathrm{Q}$ & 1.322 & $12.70 \pm 4.10$ & $>1.09 \mathrm{e}+11$ & $>5.30 \mathrm{e}+10$ & $3.20 \mathrm{e}+10$ \\
\hline $0851+202$ & $\mathrm{~B}$ & 0.306 & $15.13 \pm 0.43$ & $>4.80 \mathrm{e}+12$ & $5.08 \mathrm{e}+11$ & $2.00 \mathrm{e}+11$ \\
\hline $0906+015$ & $\mathrm{Q}$ & 1.018 & $22.08 \pm 0.47$ & $6.20 \mathrm{e}+12$ & $6.43 \mathrm{e}+11$ & $>1.40 \mathrm{e}+11$ \\
\hline $0917+624$ & $\mathrm{Q}$ & 1.446 & $12.10 \pm 1.20$ & $2.74 \mathrm{e}+11$ & $9.44 \mathrm{e}+10$ & $4.00 \mathrm{e}+10$ \\
\hline $0945+408$ & $\mathrm{Q}$ & 1.252 & $20.21 \pm 0.95$ & $>3.07 \mathrm{e}+12$ & $1.67 \mathrm{e}+11$ & $5.00 \mathrm{e}+10$ \\
\hline $0954+658$ & $\mathrm{~B}$ & 0.367 & $12.74 \pm 0.83$ & $>9.06 \mathrm{e}+11$ & $>9.06 \mathrm{e}+11$ & $>9.50 \mathrm{e}+10$ \\
\hline
\end{tabular}


Table 1.

(Continued)

\begin{tabular}{|c|c|c|c|c|c|c|}
\hline Name & Type & $z$ & $\begin{array}{c}\beta_{\mathrm{app}} \\
{[c]}\end{array}$ & $\begin{array}{c}T_{\mathrm{b}}^{15 \mathrm{GHz}, \max } \\
{[\mathrm{K}]}\end{array}$ & $\begin{array}{l}T_{\mathrm{b}}^{15 \mathrm{GHz}, 25 \% \mathrm{med}} \\
{[\mathrm{K}]}\end{array}$ & $\begin{array}{l}T_{\mathrm{b}}^{86 \mathrm{GHz}} \\
{[\mathrm{K}]}\end{array}$ \\
\hline $1012+232$ & $\mathrm{Q}$ & 0.565 & $9.00 \pm 0.60$ & $>2.48 \mathrm{e}+12$ & $4.13 \mathrm{e}+11$ & $3.90 \mathrm{e}+10$ \\
\hline $1101+384$ & B & 0.031 & $0.28 \pm 0.05$ & $2.19 \mathrm{e}+11$ & $6.14 \mathrm{e}+10$ & $2.10 \mathrm{e}+10$ \\
\hline $1128+385$ & Q & 1.733 & $1.10 \pm 0.50$ & $>4.94 \mathrm{e}+12$ & $5.30 \mathrm{e}+11$ & $8.10 \mathrm{e}+10$ \\
\hline $1150+497$ & $\mathrm{Q}$ & 0.334 & $17.50 \pm 2.00$ & $>4.94 \mathrm{e}+12$ & $5.30 \mathrm{e}+11$ & $1.40 \mathrm{e}+11$ \\
\hline $1156+295$ & $\mathrm{Q}$ & 0.729 & $24.60 \pm 1.90$ & $4.95 \mathrm{e}+12$ & $2.60 \mathrm{e}+11$ & $2.60 \mathrm{e}+11$ \\
\hline $1219+285$ & B & 0.102 & $9.12 \pm 0.79$ & $>1.43 \mathrm{e}+11$ & $>7.39 \mathrm{e}+10$ & $1.60 \mathrm{e}+10$ \\
\hline $1226+023$ & $\mathrm{Q}$ & 0.158 & $14.86 \pm 0.17$ & $>5.60 \mathrm{e}+12$ & $>4.73 \mathrm{e}+11$ & $8.50 \mathrm{e}+10$ \\
\hline $1228+126$ & $\mathrm{G}$ & 0.004 & $0.03 \pm 0.00$ & $>4.52 \mathrm{e}+11$ & $7.68 \mathrm{e}+10$ & $1.80 \mathrm{e}+10$ \\
\hline $1253-055$ & $\mathrm{Q}$ & 0.538 & $20.57 \pm 0.79$ & $>2.42 \mathrm{e}+13$ & $5.53 \mathrm{e}+12$ & $>8.90 \mathrm{e}+11$ \\
\hline $1308+326$ & $\mathrm{Q}$ & 0.997 & $27.50 \pm 1.20$ & $>2.93 \mathrm{e}+12$ & $2.26 \mathrm{e}+11$ & $1.80 \mathrm{e}+11$ \\
\hline $1502+106$ & $\mathrm{Q}$ & 1.833 & $17.55 \pm 0.90$ & $>3.22 \mathrm{e}+12$ & $>1.32 \mathrm{e}+12$ & $>2.70 \mathrm{e}+11$ \\
\hline $1508-055$ & $\mathrm{Q}$ & 1.191 & $6.20 \pm 1.20$ & $>7.84 \mathrm{e}+11$ & $3.76 \mathrm{e}+11$ & $4.60 \mathrm{e}+10$ \\
\hline $1510-089$ & $\mathrm{Q}$ & 0.360 & $28.00 \pm 0.60$ & $>5.60 \mathrm{e}+12$ & $3.20 \mathrm{e}+11$ & $>7.00 \mathrm{e}+10$ \\
\hline $1546+027$ & $\mathrm{Q}$ & 0.412 & $12.10 \pm 1.20$ & $>2.76 \mathrm{e}+12$ & $2.83 \mathrm{e}+11$ & $>2.10 \mathrm{e}+10$ \\
\hline $1548+056$ & $\mathrm{Q}$ & 1.422 & $11.60 \pm 1.70$ & $>1.03 \mathrm{e}+12$ & $3.13 \mathrm{e}+11$ & $>1.00 \mathrm{e}+11$ \\
\hline $1606+106$ & $\mathrm{Q}$ & 1.226 & $19.08 \pm 0.86$ & $>2.65 \mathrm{e}+12$ & $2.26 \mathrm{e}+11$ & $>1.50 \mathrm{e}+11$ \\
\hline $1637+574$ & $\mathrm{Q}$ & 0.751 & $13.61 \pm 0.89$ & $>1.33 \mathrm{e}+12$ & $8.54 \mathrm{e}+11$ & $3.20 \mathrm{e}+11$ \\
\hline $1642+690$ & $\mathrm{Q}$ & 0.751 & $14.53 \pm 0.26$ & $>3.19 \mathrm{e}+12$ & $>3.18 \mathrm{e}+11$ & $1.60 \mathrm{e}+11$ \\
\hline $1652+398$ & B & 0.033 & $0.87 \pm 0.21$ & $6.81 \mathrm{e}+10$ & $5.22 \mathrm{e}+10$ & $1.40 \mathrm{e}+09$ \\
\hline $1655+077$ & Q & 0.621 & $14.80 \pm 1.10$ & $>7.37 \mathrm{e}+11$ & $1.42 \mathrm{e}+11$ & $1.00 \mathrm{e}+11$ \\
\hline $1739+522$ & $\mathrm{Q}$ & 1.379 & $9.08 \pm 4.64$ & $3.77 \mathrm{e}+12$ & $1.61 \mathrm{e}+11$ & $3.90 \mathrm{e}+11$ \\
\hline $1741-038$ & $\mathrm{Q}$ & 1.057 & $6.64 \pm 1.74$ & $>2.03 \mathrm{e}+13$ & $1.35 \mathrm{e}+12$ & $1.90 \mathrm{e}+11$ \\
\hline $1749+096$ & $\mathrm{~B}$ & 0.320 & $7.90 \pm 0.75$ & $>1.85 \mathrm{e}+13$ & $>1.74 \mathrm{e}+12$ & $6.10 \mathrm{e}+11$ \\
\hline $1800+440$ & $\mathrm{Q}$ & 0.663 & $15.49 \pm 0.32$ & $>9.35 \mathrm{e}+12$ & $7.02 \mathrm{e}+11$ & $1.70 \mathrm{e}+11$ \\
\hline $1803+784$ & B & 0.680 & $10.80 \pm 1.20$ & $2.71 \mathrm{e}+12$ & $6.00 \mathrm{e}+11$ & $8.00 \mathrm{e}+10$ \\
\hline $1807+698$ & B & 0.050 & $0.09 \pm 0.01$ & $>3.92 \mathrm{e}+11$ & $1.18 \mathrm{e}+11$ & $>1.00 \mathrm{e}+11$ \\
\hline $1823+568$ & B & 0.663 & $26.20 \pm 2.60$ & $>5.34 \mathrm{e}+12$ & $>7.04 \mathrm{e}+11$ & $1.40 \mathrm{e}+11$ \\
\hline $1828+487$ & Q & 0.692 & $13.06 \pm 0.14$ & $>2.78 \mathrm{e}+12$ & $2.55 \mathrm{e}+11$ & $2.60 \mathrm{e}+10$ \\
\hline $1901+319$ & $\mathrm{Q}$ & 0.635 & $0.90 \pm 0.70$ & $7.34 \mathrm{e}+11$ & $>3.21 \mathrm{e}+11$ & $3.20 \mathrm{e}+10$ \\
\hline $1921-293$ & $\mathrm{Q}$ & 0.352 & $4.20 \pm 1.30$ & $2.75 \mathrm{e}+12$ & $4.49 \mathrm{e}+11$ & $3.90 \mathrm{e}+10$ \\
\hline $1923+210$ & $\mathrm{U}$ & $\ldots$ & $0.00 \pm 0.00$ & $2.75 \mathrm{e}+12$ & $4.49 \mathrm{e}+11$ & $5.60 \mathrm{e}+10$ \\
\hline $1928+738$ & Q & 0.303 & $8.16 \pm 0.21$ & $2.37 \mathrm{e}+12$ & $3.19 \mathrm{e}+11$ & $4.70 \mathrm{e}+10$ \\
\hline $1957+405$ & $\mathrm{G}$ & 0.056 & $0.27 \pm 0.04$ & $2.37 \mathrm{e}+12$ & $3.19 \mathrm{e}+11$ & $2.80 \mathrm{e}+10$ \\
\hline $2007+777$ & $\mathrm{~B}$ & 0.342 & $0.30 \pm 0.10$ & $>1.06 \mathrm{e}+12$ & $>1.81 \mathrm{e}+11$ & $>3.70 \mathrm{e}+11$ \\
\hline $2013+370$ & $\mathrm{~B}$ & $\ldots$ & $12.53 \pm 0.34$ & $>1.06 \mathrm{e}+12$ & $>1.81 \mathrm{e}+11$ & $1.80 \mathrm{e}+11$ \\
\hline $2037+511$ & $\mathrm{Q}$ & 1.687 & $3.78 \pm 0.53$ & $8.33 \mathrm{e}+11$ & $6.07 \mathrm{e}+11$ & $4.20 \mathrm{e}+11$ \\
\hline $2121+053$ & $\mathrm{Q}$ & 1.941 & $11.64 \pm 0.74$ & $>1.24 \mathrm{e}+13$ & $2.63 \mathrm{e}+12$ & $8.70 \mathrm{e}+10$ \\
\hline $2128-123$ & $\mathrm{Q}$ & 0.501 & $6.00 \pm 0.62$ & $>3.37 \mathrm{e}+11$ & $>1.29 \mathrm{e}+11$ & $2.30 \mathrm{e}+09$ \\
\hline $2134+004$ & $\mathrm{Q}$ & 1.932 & $5.07 \pm 0.32$ & $>1.31 \mathrm{e}+12$ & $1.97 \mathrm{e}+11$ & $>4.60 \mathrm{e}+10$ \\
\hline $2155-152$ & $\mathrm{Q}$ & 0.672 & $18.10 \pm 1.80$ & $>8.77 \mathrm{e}+11$ & $>6.53 \mathrm{e}+11$ & $2.00 \mathrm{e}+10$ \\
\hline $2200+420$ & $\mathrm{~B}$ & 0.069 & $9.95 \pm 0.72$ & $2.26 \mathrm{e}+12$ & $>5.81 \mathrm{e}+11$ & $>5.50 \mathrm{e}+12$ \\
\hline $2201+315$ & $\mathrm{Q}$ & 0.298 & $8.28 \pm 0.10$ & $>2.77 \mathrm{e}+12$ & $>1.06 \mathrm{e}+11$ & $5.00 \mathrm{e}+10$ \\
\hline $2216-038$ & $\mathrm{Q}$ & 0.901 & $6.75 \pm 0.70$ & $7.04 \mathrm{e}+11$ & $>1.05 \mathrm{e}+11$ & $5.80 \mathrm{e}+09$ \\
\hline $2223-052$ & $\mathrm{Q}$ & 1.404 & $20.33 \pm 0.65$ & $>6.53 \mathrm{e}+12$ & $>1.42 \mathrm{e}+12$ & $7.30 \mathrm{e}+10$ \\
\hline $2234+282$ & $\mathrm{Q}$ & 0.795 & $5.10 \pm 2.20$ & $>5.13 \mathrm{e}+11$ & $4.20 \mathrm{e}+10$ & $4.40 \mathrm{e}+10$ \\
\hline $2251+158$ & $\mathrm{Q}$ & 0.859 & $13.79 \pm 0.49$ & $>3.37 \mathrm{e}+12$ & $4.20 \mathrm{e}+11$ & $>1.30 \mathrm{e}+11$ \\
\hline $2255-282$ & $\mathrm{Q}$ & 0.927 & $6.00 \pm 0.95$ & $>2.24 \mathrm{e}+13$ & $4.93 \mathrm{e}+12$ & $2.00 \mathrm{e}+10$ \\
\hline $2345-167$ & $\mathrm{Q}$ & 0.576 & $11.46 \pm 0.76$ & $>1.39 \mathrm{e}+12$ & $2.80 \mathrm{e}+11$ & $1.10 \mathrm{e}+09$ \\
\hline
\end{tabular}




\subsection{Constraining Intrinsic Brightness Temper- ature}

Fig. 2 shows plots of the apparent speed $\beta_{\text {app }}$ versus the observed brightness temperature $T_{\mathrm{b}}$ for our sample. The top panel is a plot of $\beta_{\text {app }}$ versus the maximum observed brightness temperatures at $15 \mathrm{GHz}$ for each source in our sample. The dashed line indicates sources at the critical angle which have $T_{0}=1.2 \times 10^{11} \mathrm{~K}$, and the solid grey line was calculated using the same value for $T_{0}$. The value of $T_{0}$ was chosen to have about $75 \%$ of the sources in the right of and below the dashed line, which is the same criterion for choosing the value of $T_{0}$ as in Homan et al. (2006). The middle panel is for sources in their median-low ( $25 \%$ median) state. This is the median of the lowest half of the brightness temperature observations for a given source as defined in Homan et al. (2006). The $25 \%$ median determined in this critera represents a typical low brightness state for each source at $15 \mathrm{GHz}$. We found a characteristic intrinsic brightness temperature of $T_{0}=2.0 \times 10^{10} \mathrm{~K}$ for the $25 \%$ median state. The intrinsic temperatures chosen at $15 \mathrm{GHz}$ for our sample (98 sources) are very close to those for the sample (106 sources) of Homan et al. (2006). This implies that two samples are statistically similar and hence suitable for applying this method.

The bottom panel of Fig. 2 is a similar plot for the observed brightness temperatures at $86 \mathrm{GHz}$ for each source in our sample. The value of $T_{0}=4.8 \times 10^{9} \mathrm{~K}$ was chosen to have about $75 \%$ of the sources in the right of and below the dashed line. Since other choices for the simulation parameters described in Homan et al. (2006) give a very similar distribution of $\beta_{\text {app }}$ vs $T_{\mathrm{b}}$ and have fractions between $60 \%$ and $80 \%$ of sources with their viewing angles smaller than the critical angle, we take the corresponding values of $T_{0}=3.3 \times 10^{9} \mathrm{~K}$ for a $80 \%$ fraction and $T_{0}=7.4 \times 10^{9} \mathrm{~K}$ for a $60 \%$ fraction as the range of uncertainty for $T_{0}$.

Fig. 3 shows plots similar to Fig. 2 with sources divided into three groups: quasars, BL Lac objects, and galaxies. Galaxies have lower apparent jet speeds, whereas quasars and BL Lac objects are widely spread in speed.

\subsection{Doppler Factor}

The narrow range of intrinsic brightness temperatures determined at $15 \mathrm{GHz}$ (median-low state) and $86 \mathrm{GHz}$ enables us to derive Doppler factors for the sources in our sample according to Eq. 3. Figs. 4a and $4 \mathrm{~b}$ show the distributions of estimated Doppler factors for the $15 \mathrm{GHz}$ (25\% median) data and the $86 \mathrm{GHz}$ data. The distributions have the mean values of 31.2 and 24.1 (excluding one value exceeding 1000) with the medians of 16.1 and 14.6 for each data, respectively. This implies that Doppler factors derived with the data at $15 \mathrm{GHz}$ and $86 \mathrm{GHz}$ for our sample of sources are slightly different from each other. The Doppler factors of the VLBI core at $86 \mathrm{GHz}$ are lower than those
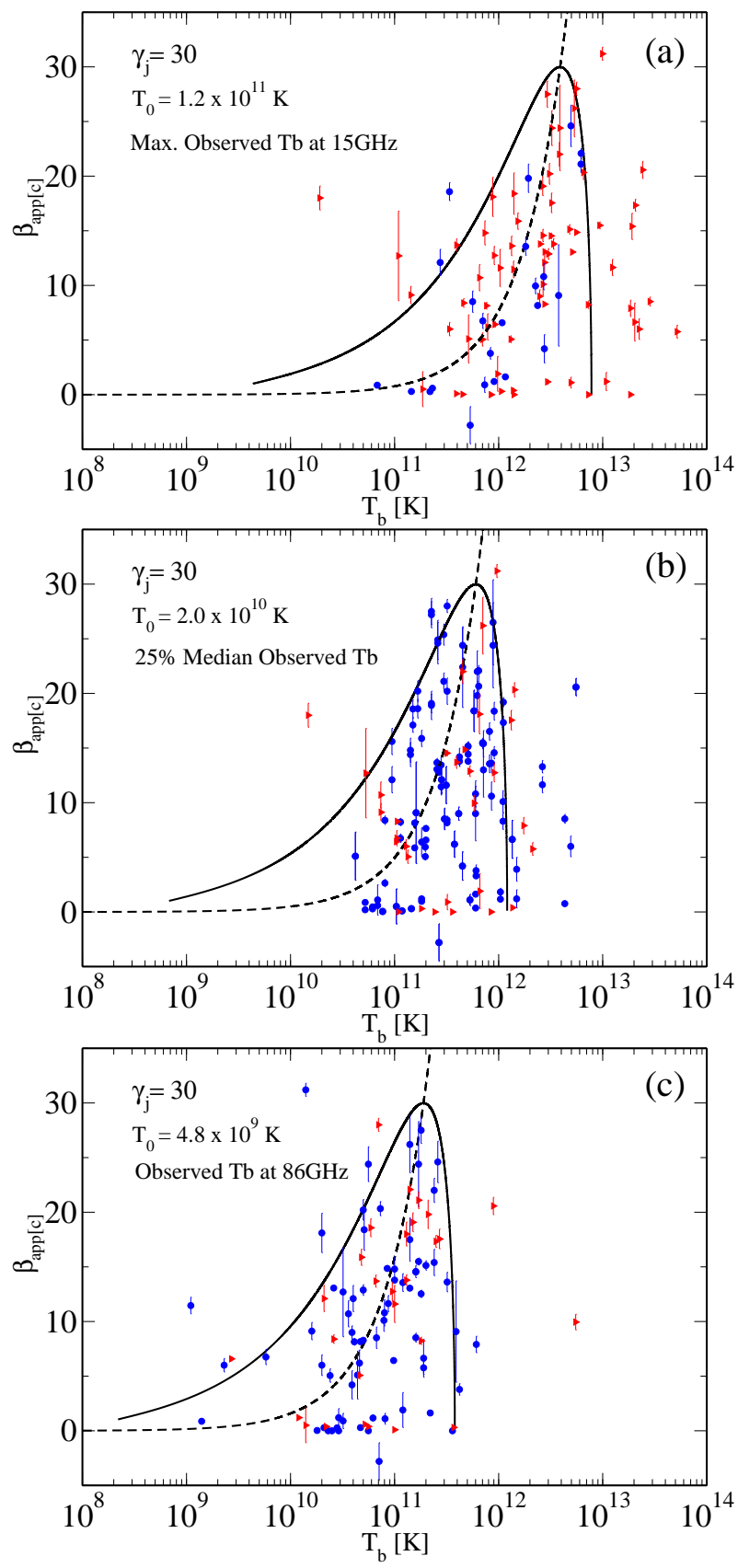

Fig. 2.- Plots of apparent jet speed versus observed brightness temperature for the sources in our sample. (a) and (b) plots are for the $15 \mathrm{GHz}$ data. (c) plot is for the $86 \mathrm{GHz}$ data. Lower limits of brightness temperatures are indicated by right triangle, and solid circles represent measurements. The dashed line represents sources observed at the critical angle that have the intrinsic brightness temperature of $T_{0}=1.2 \times 10^{11} \mathrm{~K}(\mathrm{a}), T_{0}=2.0 \times 10^{10} \mathrm{~K}(\mathrm{~b})$, and $T_{0}=4.8 \times 10^{9} \mathrm{~K}(\mathrm{c})$. The solid grey line represents the possible apparent speeds of a $\gamma_{\mathrm{j}}=30$ source with the corresponding intrinsic brightness temperatures. 

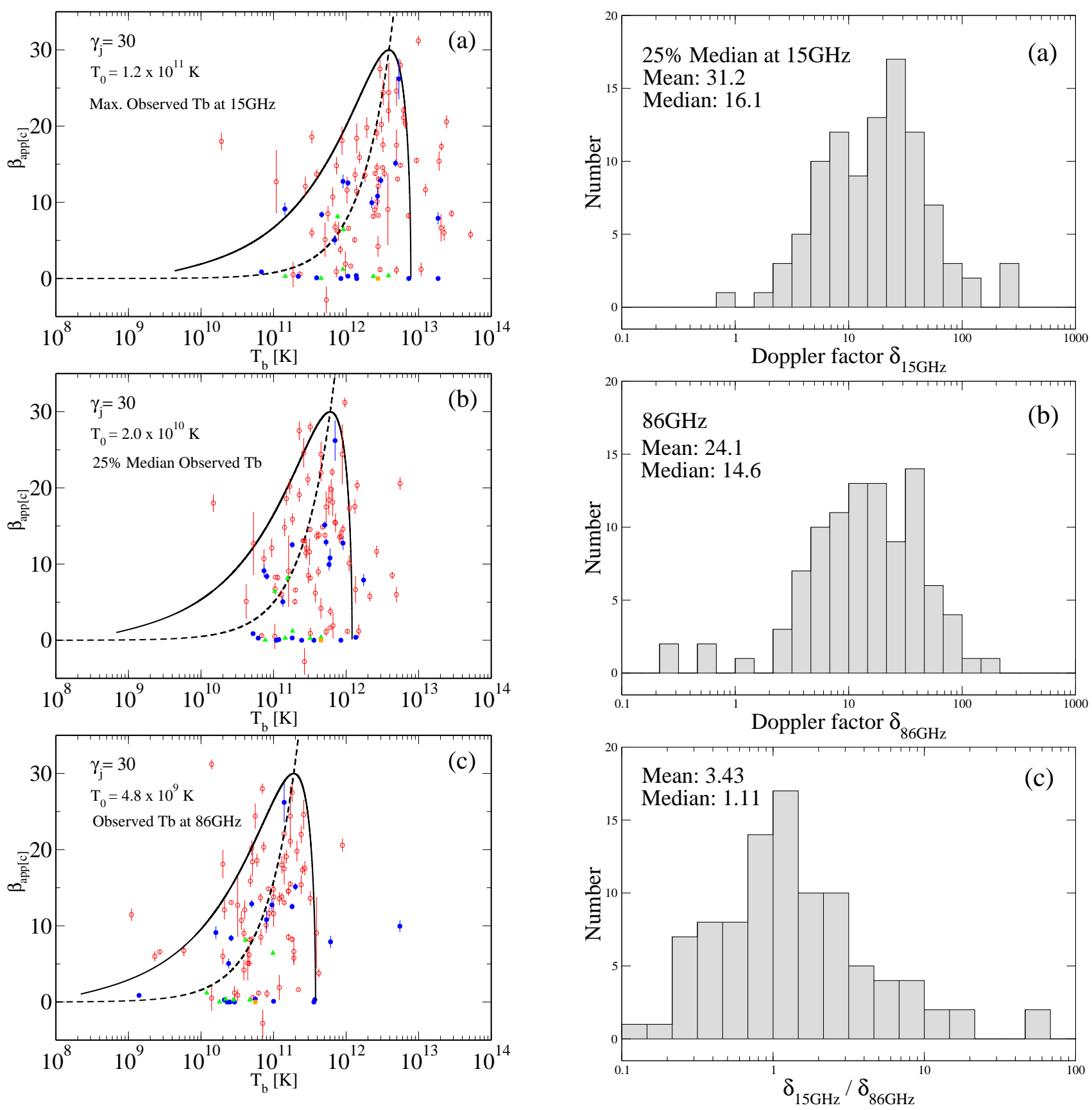

Fig. 3.- Similar plots as Fig. 2 except for the symbols: red open circles represent quasars; blue solid circles represent BL Lac objects; green triangles represent galaxies; one orange square represents unidentified source.

at $15 \mathrm{GHz}$. Fig. 4c shows the distribution of the ratio of Doppler factors at $15 \mathrm{GHz}$ and $86 \mathrm{GHz}$ for all sources in our sample. The distribution peaks at a value higher than unity, and has mean and median values of 3.43 and 1.11, implying that for many sources the estimated Doppler factors are higher for the $15 \mathrm{GHz}$ jets than for the $86 \mathrm{GHz}$ VLBI cores. Taking into account Eq. 4, higher Doppler factors indicate faster ap-

Fig. 4. - Distributions of Doppler factors at (a) $15 \mathrm{GHz}$ and (b) $86 \mathrm{GHz}$, and of (c) their ratio. Mean and median values of each distribution are shown in each plot.

parent jet speeds for sources whose viewing angles are close to the critical value $\theta_{\mathrm{c}}$ for maximal apparent jet speed.

\section{DISCUSSION}

It is interesting that the plots $\beta_{\mathrm{app}}$ versus $T_{\mathrm{b}}$ in Figs. $2 \mathrm{~b}$ and $2 \mathrm{c}$ show similar trends although few 
sources are far beyond the solid curve in Fig. 2c. There are few sources having low brightness and fast apparent speeds. As discussed in Cohen et al. (2007), this implies that for the fastest jet components in many sources, the pattern speeds are closely related with the flow speeds. If the pattern and flow speeds were independent, then it is difficult to imagine such a trend in $\beta_{\text {app }}$ versus $T_{\mathrm{b}}$. If some sources have fast pattern speeds with slow flow speeds, then they may be placed to the left of the solid line. This may be the case for the few sources located in the area of Fig. 2c.

As discussed in Homan et al. (2006), the chosen value of $T_{0}$ should be considered as a lower bound on the characteristic value. This is because there are more lower limits of observed brightness temperatures than measurements in Fig. 2a. For our sample $T_{0}>1.2 \times 10^{11} \mathrm{~K}$ when sources have their maximum brightness temperature. Therefore sources should be well away from equipartition. However, for the medianlow state in Fig. 2b, there are fewer limits of the observed temperatures than measurements. We may consider the chosen value of $T_{0}$ the characteristic medianlow intrinsic brightness temperature. For our sample $T_{0}=2.0 \times 10^{10} \mathrm{~K}$. This value of $T_{0}$ is similar to the brightness temperature under an equipartition condition.

For the $86 \mathrm{GHz}$ data, there are similar number of lower limits as the median-low plot, so it seems reasonable to take the characteristic intrinsic brightness temperature to be $T_{0}=4.8 \times 10^{9} \mathrm{~K}$. This value is lower by a factor of $\sim 4$ than the median-low intrinsic brightness temperature at $15 \mathrm{GHz}$. We note that the apparent decrease in brightness temperature between the $15 \mathrm{GHz}$ and $86 \mathrm{GHz}$ cases cannot be attributed to scatter in the plots, since the real ranges of the intrinsic brightness temperate in both cases are $1.6 \times 10^{10} \mathrm{~K}<T_{0}<$ $3.1 \times 10^{10} \mathrm{~K}$ and $3.3 \times 10^{9} \mathrm{~K}<T_{0}<7.4 \times 10^{9} \mathrm{~K}$ for the $15 \mathrm{GHz}$ and $86 \mathrm{GHz}$ cases, respectively, using the $60 \%$ - and $80 \%$-fraction criteria.

This is lower-than-equipartition temperature implying that the VLBI cores seen at $86 \mathrm{GHz}$ may be representing a jet region where the magnetic field energy dominates the total energy in the jet. Using Eq. 5 of Readhead (1994), we estimate that the $86 \mathrm{GHz}$ VLBI core regions have $4.5 \times 10^{8}$ times more energy in magnetic fields as in radiating particles. In these circumstances we may expect conversion of magnetic field energy into the kinetic energy of particles in the jet. Since the $86 \mathrm{GHz}$ VLBI cores should be located the inner regions of the jet in contrast to the $15 \mathrm{GHz}$ cores and jets, taking into account the opacity effect of a relativistic jet (Lobanov 2007; Lobanov \& Zensus 2006), we may also expect that the intrinsic brightness temperature will increase as we go down-stream of the jet. Applying similar estimates to the $15 \mathrm{GHz}$ data, we found $1 / \eta \simeq 2.4 \times 10^{3}$, implying the ratio between the energy in the magnetic fields and in their radiating particles may change by an order of 5 as the relativistic jet moves from the $86 \mathrm{GHz}$ VLBI core regions to the $15 \mathrm{GHz}$ jet regions.

The increase of the intrinsic brightness temperature may result in the increase of apparent jet speeds from the jet core of radio galaxies and BL Lac objects as reported by Lister et al. (2013). Fig. 13 of Lister et al. (2013) may imply that the apparent jet speeds at $15 \mathrm{GHz}$ (in the outer region of the jet) are faster than those at $86 \mathrm{GHz}$ (in the inner region), which is consistent with our results of Doppler factor in Section 3.4. Although positive correlation of speed with core distance needs to be confirmed based on a complete AGN sample, we may expect that the relativistic jets of AGNs may accelerate on moving away from the central engine with corresponding increase in the intrinsic brightness temperature. This also agrees with the results of Doppler factors. Some observational tests for the jet acceleration model will be discussed in a separate paper.

The difference in the intrinsic temperatures $T_{0}$ deduced at $15 \mathrm{GHz}$ and $86 \mathrm{GHz}$ may imply that only a small number of sources will be suitable for VLBI at higher frequencies (e.g., $\geq 300 \mathrm{GHz}$ ).

\section{CONCLUSIONS}

- The $86 \mathrm{GHz}$ global VLBI survey has yielded the observed brightness temperatures for 98 sources with available apparent jet speeds and observed brightness temperatures at $15 \mathrm{GHz}$ from the $2 \mathrm{~cm}$ VLBA survey and MOJAVE program. On applying the $T_{0}$-constraining method, we find that the intrinsic brightness temperature is $T_{0}=4.8_{-1.5}^{+2.6} \times$ $10^{9} \mathrm{~K}$ for the VLBI cores seen at $86 \mathrm{GHz}$.

- The Doppler factors estimated with the constrained intrinsic brightness temperatures tend to be higher for the jets seen at $15 \mathrm{GHz}$ than for the VLBI cores seen at $86 \mathrm{GHz}$. It is likely that Doppler factor increases down-stream of a relativistic jet.

- The VLBI cores at $86 \mathrm{GHz}$ in our sample may be such that the magnetic field energy in the jet converts into the kinetic energy of particles. Moving outwards, down stream of the jet, the intrinsic brightness temperature will increase.

\section{ACKNOWLEDGMENTS}

I would like to thank the anonymous referee for important comments and suggestions which have enormously improved the manuscript. The VLBA is an instrument of the National Radio Astronomy Observatory, which is a facility of the National Science Foundation operated under cooperative agreement by Associated Universities, Inc.. This work was supported by Global Research Collaboration and Networking program of Korea Research Council of Fundamental Science \& Technology (KRCF). 


\section{REFERENCES}

Abdo, A. A., Ackermann, M., Ajello, M., Axelsson, M., Baldini, L., Ballet, J., Barbiellini, G., Bastieri, D., Baughman, B. M., Bechtol, K., et al. 2010, A Change in the Optical Polarization Associated with a $\gamma$-Ray Flare in the Blazar 3C279, Nature, 463, 919

Blandford, R. D., \& Königl, A. 1979, Relativistic Jets as Compact Radio Sources, ApJ, 232, 34

Burbidge, G. R., \& Burbidge, E. M. 1957, The Sources of Radio Emission in NGC 5128 and NGC 1316, ApJ, 125, 1

Cohen, M. H., Lister, M. L., Homan, D. C., Kadler, M., Kellermann, K. I., Kovalev, Y. Y., \& Vermeulen, R. C. 2007, Relativistic Beaming and the Intrinsic Properties of Extragalactic Radio Jets, ApJ, 658, 232

Croston, J. H., Hardcastle, M. J., Harris, D. E., Belsole, E., Birkinshaw, M., \& Worrall, D. M. 2005, An XRay Study of Magnetic Field Strengths and Particle Content in the Lobes of FR II Radio Sources, ApJ, 626,733

Homan, D. C., Lister, M. L., Kellermann, K. I., Cohen, M. H., Ros, E., Zensus, J. A., Kadler, M., \& Vermeulen, R. C. 2003, Jet Collimation in Action: Realignment on Kiloparsec Scales in 3C 279, ApJ, 589, L9

Homan, D. C., Kovalev, Y. Y., Lister, M. L., Ros, E., Kellermann, K. I., Cohen, M. H., Vermeulen, R. C., Zensus, J. A., \& Kadler, M. 2006, Intrinsic Brightness Temperatures of AGN Jets, ApJ, 642, L115

Hughes, P. A., \& Miller, L. 1991, Introduction: Synchrotron and Inverse-Compton Radiation, Beams and Jets in Astrophysics, 1

Kellermann, K. I., \& Pauliny-Toth, I. I. K. 1985, The Spectra of Opaque Radio Sources, ApJ, 295, 358

Kellermann, K. I., Lister, M. L., Homan, D. C., Vermeulen, R. C., Cohen, M. H., Ros, E., Kadler, M., Zensus, J. A., \& Kovalev, Y. Y. 2004, Sub-Milliarcsecond Imaging of Quasars and Active Galactic Nuclei. III. Kinematics of Parsec-Scale Radio Jets, ApJ, 609, 539

Kovalev, Y. Y., Kellermann, K. I., Lister, M. L., Homan, D. C., Vermeulen, R. C., Cohen, M. H., Ros, E., Kadler, M., Lobanov, A. P., Zensus, J. A., Kardashev, N. S., Gurvits, L. I., Aller, M. F., \& Aller, H. D. 2005, Sub-Milliarcsecond Imaging of Quasars and Active Galactic Nuclei. IV. Fine-Scale Structure, AJ, 130, 2473

Lee, S.-S., Lobanov, A. P., Krichbaum, T. P., Witzel, A., Zensus, J. A., Bremer, M., Greve, A., \& Grewing, M. 2008, A Global $86 \mathrm{GHz}$ VLBI Survey of Compact Radio Sources, AJ, 136, 159

Lind, K. R., \& Blandford, R. D. 1985, Semidynamical Models of Radio Jets - Relativistic Beaming and Source Counts, ApJ, 295, 358
Lister, M. L., Cohen, M. H., Homan, D. C., Kadler, M., Kellermann, K. I., Kovalev, Y. Y., Ros, E., Savolainen, T., \& Zensus, J. A. 2009, MOJAVE: Monitoring of Jets in Active Galactic Nuclei with VLBA Experiments. VI. Kinematics Analysis of a Complete Sample of Blazar Jets, AJ, 138, 1874

Lister, M. L., Aller, M. F., Aller, H. D., Homan, D. C., Kellermann, K. I., Kovalev, Y. Y., Pushkarev, A. B., Richards, J. L., Ros, E., \& Savolainen, T. 2013, MOJAVE. X. Parsec-Scale Jet Orientation Variations and Superluminal Motion in AGN, AJ, in press (arXiv:1308.2713)

Lobanov, A. P. 1998, Ultracompact Jets in Active Galactic Nuclei, A\&A, 330, 79

Lobanov, A. P., \& Zensus, J. A. 2006, Extragalactic Relativistic Jets and Nuclear Regions in Galaxies, astro-ph/0606189

Lobanov, A. P. 2007, Compact Jets as Probes for SubParsec Scale Regions in AGN, Ap\&SS, 311, 263

Marscher, A. P. 1990, Interpretation of Compact Jet Observations, Parsec-scale radio jets, ed. Zensus \& Peason (Cambridge: Cambridge University Press), 236

Marscher, A. P. 1995, Probes of the Inner Jets of Blazars, Proceedings of the National Academy of Science, 92, 11439

Marscher, A. P. 2006, Relativistic Jets in Active Galactic Nuclei, AIP Conf. Proc. 856: Relativistic Jets: The Common Physics of AGN, Microquasars, and Gamma-Ray Bursts, 1

Véron-Cetty, M.-P., \& Véron, P. 2006, A Catalogue of Quasars and Active Nuclei: 12th Edition, A\&A, 455,773

Readhead, A. C. S. 1994, Equipartition Brightness Temperature and the Inverse Compton Catastrophe, ApJ, 426, 51 